\title{
Strain Membrane Design of SOI Pressure Sensor
}

\author{
Jun.Zhao ${ }^{1, a}$, Zhi.Jun. Yao, b and Zheng. $\mathrm{Li}^{3, \mathrm{c} *}$ \\ ${ }^{1}$ National-Provincial Laboratory of Special Function Thin Film Materials, and School of Materials \\ Science and Engineering, Xiangtan University, Hunan 411105, China \\ ${ }^{2}$ Center for Semiconductor Particle and photon Imaging Detector Development and Fabrication, \\ Xiangtan University, Xiangtan 411105, China \\ azj422670934@163.com, byzj791582465@126.com, 'czhengli58@gmail.com
}

Keywords: SOI pressure sensor; the strain membrane; the pressure sensitive resistance; sensitivity;

Abstract: The SOI pressure sensor is a new high temperature pressure sensor made of SOI materials. The sensitivity of the SOI pressure sensor is determined by the design of the strain membrane and the pressure sensitive resistance. In this paper, the optimal size and thickness of the strain membrane, and the position of the doped pressure sensitive resistor are obtained by analyzing simulation results of chip strain membranes with the different sizes using a simulation tool ANSYS. These results can provide an important scientific basis to the SOI pressure sensor chip design.

\section{Introduction}

In the development of SOI pressure sensor, the design of strain membrane and pressure sensitive resistor is the core part. The main factors that affect the strain membrane are the strain membrane size and the position of pressure sensitive resistor.

Shapes of circular, square and rectangular are generally used in designing the strain membrane. In order to improve the linearity and sensitivity of the output signals, new design concepts such as single island, double island and beam-membrane-island membrane have been emerged in recent years $^{[1]-[2]}$. The square membrane is superior to the circular membrane. With the same membrane size, the maximum stress on the square membrane can be 1.64 times of that of the circular membrane ${ }^{[3]}$.

In SOI pressure sensor, the relative change of the pressure sensitive resistance is:

$$
\frac{\Delta R}{R}=\frac{\pi_{44}}{2}\left|\sigma_{l}-\sigma_{t}\right|
$$

Where $\pi_{44}$ is the tangential pressure sensitive resistance coefficient, $\sigma_{l}$ is the longitudinal stress, $\sigma_{t}$ is the transverse stress. From Eq. 1, we can know that the maximum value of $\left|\sigma_{l}-\sigma_{t}\right|$ determines the relative change of the pressure sensitive resistor. The pressure sensitive resistor will be more sensitive when the value of $\max \left|\sigma_{l}-\sigma_{t}\right|$ is larger.

The design of strain membrane and the position of pressure sensitive resistor are mainly described in this paper. The sensor chip performance is simulated by finite element analysis software ANSYS with the thin plate of strain mechanics theory. five finite element models of chip structure are established with different parameters of sensor chip structure and material. The stress distribution on the chip is simulated by adding pressure loads in a specific range and boundary constraints. The position of pressure sensitive resistor is obtained by comparing the $\max \left|\sigma_{1}-\sigma_{t}\right|$ at different regions of membrane. 


\section{The strain membrane length-width ratio dependence}

The effect of length-width ratio on the SOI pressure sensor sensitivity is studied in this part. Keeping the strain membrane area fixed to $10^{6} \mu \mathrm{m}^{2}$ and the strain membrane thickness fixed to 30 $\mu \mathrm{m}$, five finite element models of chip structure are simulated when the length-width ratio is changing from $5: 1,4: 1,3: 1,2: 1$, and $1: 1$ to find out the maximize output of $\frac{\Delta R}{R}$ and the corresponding strain membrane length-width ratio. After data processing, the longitudinal and transverse stressed on $\mathrm{X}$ and $\mathrm{Y}$ axes with different strain membrane length-width ratios are displayed in Fig. 1. As can be seen from Fig. 1, there is a decline in the theoretical output value of the sensor as the width-length ratio increase when the membrane area is fixed. Therefore, it is better to use the square membrane in order to get the maximum theoretical output since the theoretical output value is maximum when the ratio is $1: 1$.

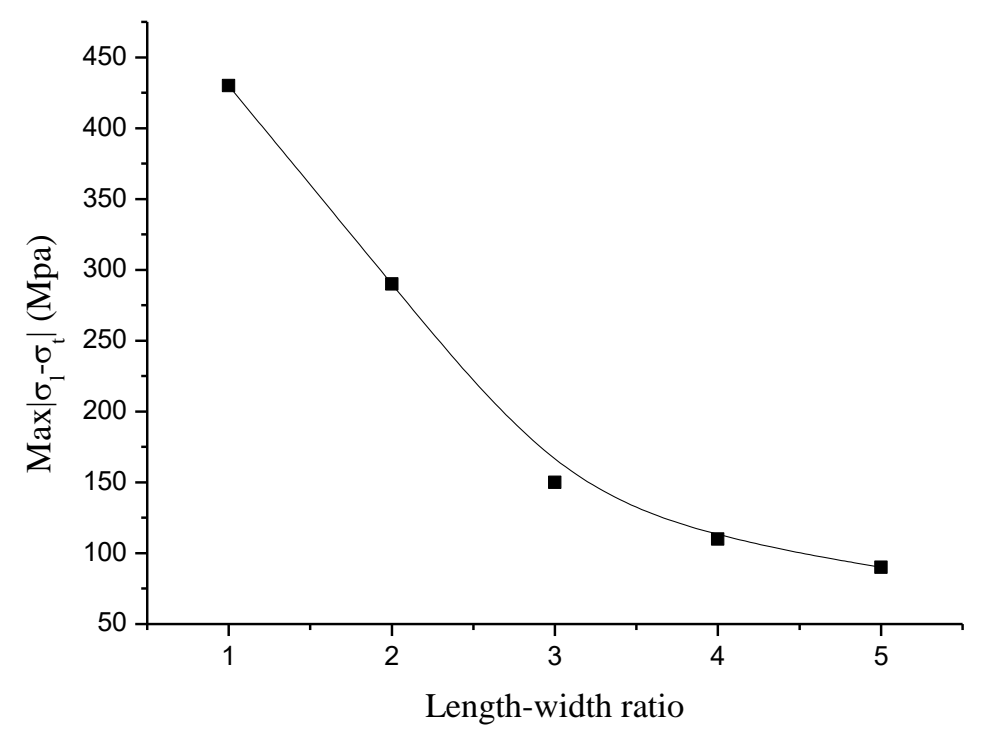

Fig. 1 value of $\max \left|\sigma_{1}-\sigma_{t}\right|$ for sensor of different width-length ratio

\section{The strain membrane thickness dependence}

The stress and pressure will not be linear when the formation in membrane center is larger than the thickness of the membrane. Usually we keep the maximum deformation less than $20 \%$ of the membrane thickness to obtain good linear output.

The membrane just bends and doesn't stretch in small deformation case. The deformation is maximum at the membrane center and the value of it $\left(\omega_{\max }\right)$ is ${ }^{[5]}$ :

$$
\omega_{\max }=0.01518 \frac{P A^{4}\left(1-v^{2}\right)}{E H^{2}} \leq \frac{1}{5} H
$$

Where $E$ is the strain modulus of silicon( $\sim 190 \mathrm{GPa}), v$ is the poisson's $\operatorname{ratio}(\sim 0.278), A$ is the strain membrane side length, $P$ is the pressure load, $H$ is the strain membrane thickness.

We can calculate the membrane thickness of $H \geq 29.3 \mu \mathrm{m}$, if $P$ at $2 \mathrm{MPa}$ and $A$ is $500 \mu \mathrm{m}$. Since we use the strict symmetric square shape membrane, we only need to take the longitudinal and transverse stresses on the $\mathrm{X}$ axis. The calculated the $\max \left|\sigma_{l}-\sigma_{t}\right|$ as a function of membrane thickness with fixed area is shown in Fig. 2. 


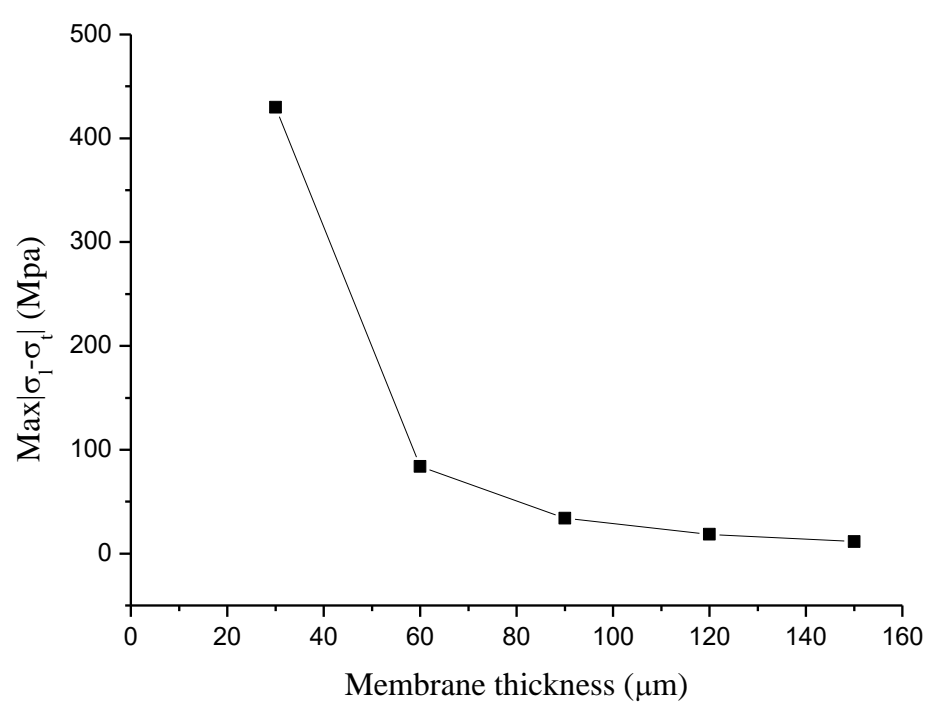

Fig. 2 value of $\max \left|\sigma_{l}-\sigma_{t}\right|$ for sensor of different membrane thickness

As can be seen from the Fig. 2, when the membrane area is fixed, the theoretical output value of the sensor is declines exponentially with the membrane thickness. So, the thinnest membrane, while maintaining the membrane mechanical integrally to get the maximum theoretical output of $\frac{\Delta R}{R}$.

\section{Design the location of electrical bridge resistance}

According to the conclusions above, we can set the following parameters of the sensor pressure sensor for calculations: sensor thickness $H_{l}=400 \mu \mathrm{m}$; sensor side length $L=2000 \mu \mathrm{m}$; the strain membrane thickness $H=30 \mu \mathrm{m}$; the strain membrane side length $A=1000 \mu \mathrm{m}$. As mentioned before, due to symmetry, we only need to simulate on quarter of the sensor chip, Also, as show in Fig. 3, we set surface constrains on boundaries X, Y and Z surface. Adding a pressure load $P=2 \mathrm{MPa}$ on the strain membrane. Fig. 4 shows the deformation of the strain membrane of a pressure sensor under an uniform load. The results show the stress distribution of Von Mises, which is an equivalent $\operatorname{stress}(\sigma)$ based on shear strain energy:

$$
\sigma=\left[\frac{\left(\sigma_{1}-\sigma_{2}\right)^{2}+\left(\sigma_{2}-\sigma_{3}\right)^{2}+\left(\sigma_{3}-\sigma_{1}\right)^{2}}{2}\right]^{1 / 2}
$$

Where $\sigma_{\mathrm{i}(\mathrm{i}=1,2,3)}$ represents the ith principal stresses.

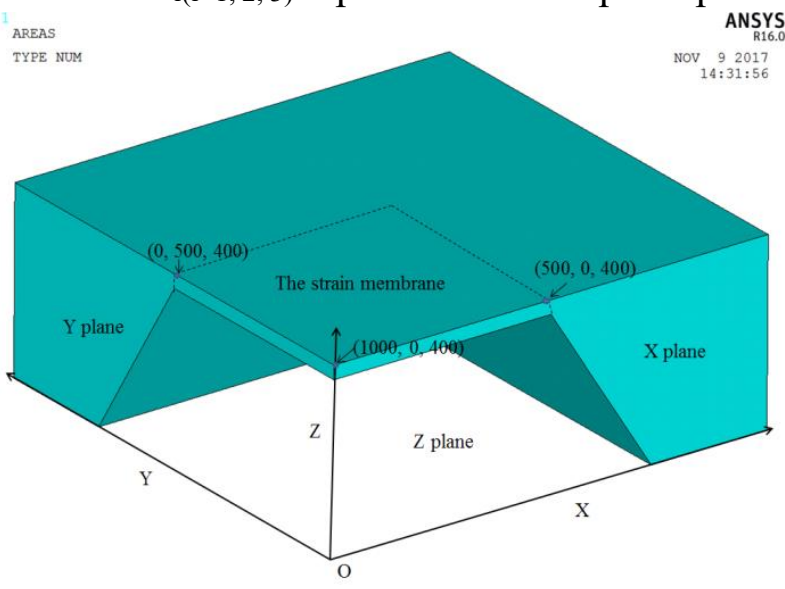

Fig. 3 The structure of SOI pressure sensor

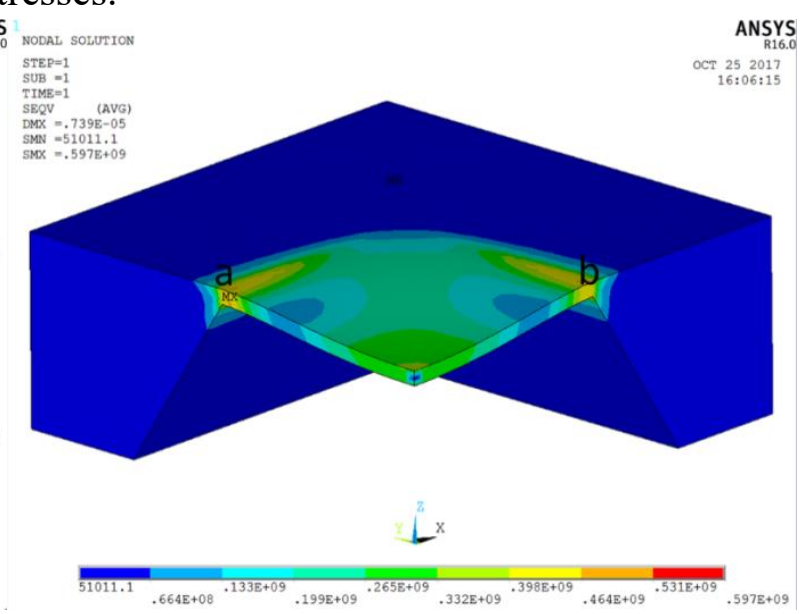

Fig. 4 Von Mises stress distribution 
From Fig. 4 we can also see that, when the membrane is under an uniform load, maximum stresses are at point "a" and point "b" near edges of the membrane.

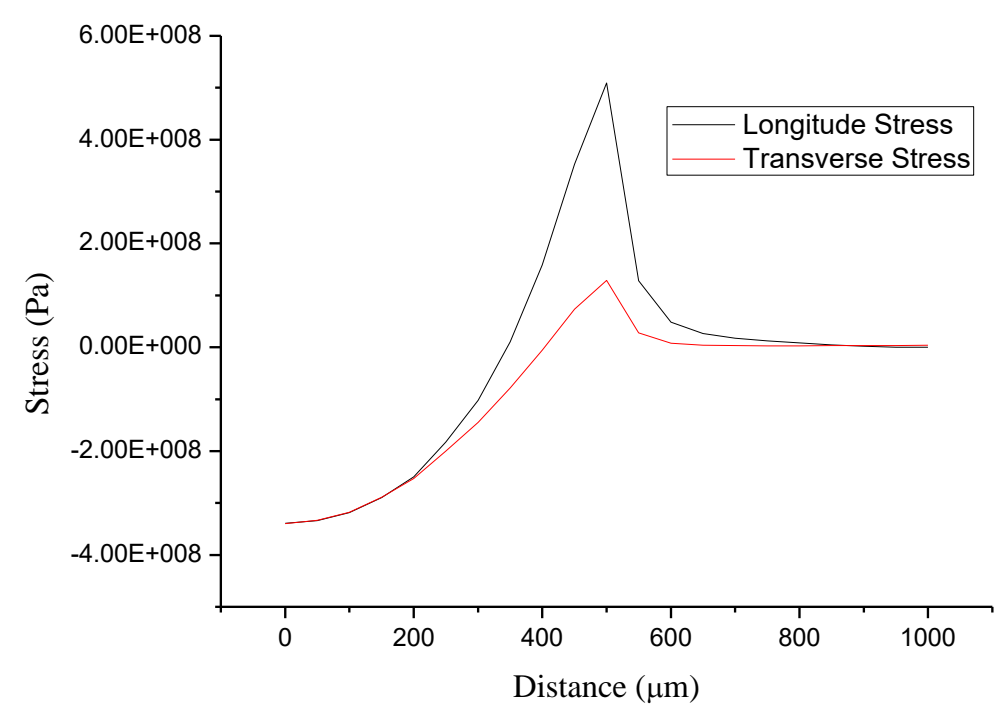

Fig. 5 The longitudinal and transverse stresses distribution in the $\mathrm{X}$ direction

From Eq. 1, we know that the difference between the longitudinal and transverse stresses determines the sensitivity of the sensor. We define a path " $\mathrm{XG}$ " along the $\mathrm{X}$ axis (or $\mathrm{Y}$ axis, it does not matter since the membrane is a square) from the center of membrane $(0,0,400)$ to the edge of the membrane $(1000,0,400)$. The longitudinal and transverse stresses along the path are shown in Fig. 5. The plot is smooth thus the mesh is fine enough for the simulation to accurately reflect the actual situation. Ignoring the calculation error caused by meshing, when $\mathrm{X}$ coordinate value is in [0, 250] along " $X G$ ", longitudinal and transverse stresses are almost equal, indicating the sensitivity in the central region of the strain membrane is approximately 0 . The difference between the longitudinal and transverse stresses is not significant when $X$ coordinate value is in $[250,480]$ along "XG". So, the pressure sensitive resistor should not be placed in the $X$ interal $[0,480]$, The difference between the longitudinal and transverse stress is maximum when $\mathrm{X}$ coordinate value is 500 , so the maximum sensitivity will be obtained by placing four pressure sensitive resistors at $(0$, $-500,400),(0,500,400),(500,0,400)$ and $(-500,0,400)$. Fig. 4 is the stress simulation of just $1 / 4$ of the membrane.

The four resistors that make up the bridge arm must meet the following requirements:

(1) The stress of each resistor is set as large as possible and the value of the stress must be equal for four resistors.

(2) Each resistor must have the same pressure sensitive resistance coefficient, and the longitudinal and transverse coefficient of each pressure resistor must be opposite to each other. Therefore two of the four pressure-sensitive resistors are parallel to $<110>$ direction and the other two are parallel to $<1 \overline{10}>$ direction (the strain membrane is the (100) plane).

According to these requirements, each identical resistor is placed on the edge of the strain membrane, at $500 \mu \mathrm{m}$ from the membrane center. We design a resistor layout plan as shown in Fig. 6. 


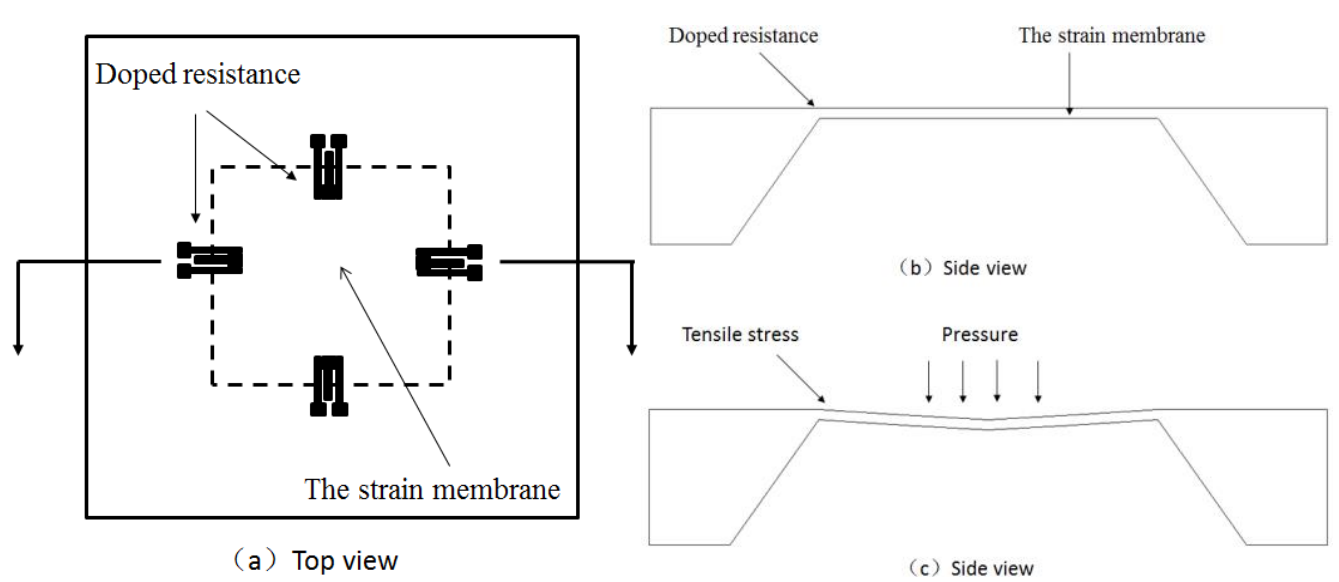

Fig. 6 the resistor layout plan

\section{Conclusion}

Using the ANSYS finite element analysis software, the stress distribution on the membrane surface of pressure sensor is simulated. Dependence of the sensor performance on the strain membrane length-width ratio and thickness is systematically analyzed. The sensor sensitivity, has been found to increase as the membrane thickness decreases. While the measuring range of sensor increases with membrane thickness. Our work shows that when the range is $2 \mathrm{MPa}$, the optimum strain membrane length-width ratio is $1: 1$, and the optimum membrane thickness is $30 \mu \mathrm{m}$. Optimization layout scheme of strain resistors is also given according to the stress distribution. The simulation and analysis provide reliable insights to the design and the fabrication of SOI pressure sensors.

\section{References}

[1] SNDMAIWER H, KOHL K. Piezoresistibive low-pressure sensor with high sensitivity and high accuracy[J]. Sensors and Actuators, 1990, 1121 - 23:142 - 145.

[2] BAO M, YU L, WANG Y. Micromechanical beam-diaphragm structure improves performances of pressure transducers[J]. Sensors and Actuators, 1990, $1121-23: 137-141$.

[3] HEIN S, SCHLICHTING V, OBERMEIER E. Pirzoresistive silicon sensor for very low pressure based on concept of stress concentration[C] // Int Conf on Solid-State Sensors and Actuators (Traps ducers93) Yokohama, Japan, 1993: 628 - 631.

[4] Yozo Kanda, Akio Yasukawa. Optimum design considerations for silicon piezoresistive pressure sensors, sensors and actuators, 1997, A 62: 539 - 542

[5] Ying Zeng, Li Ren Yan, Ji Ming Wang. Microelectronics Manufacturing Scientific Principles[M]. BeiJing: Electronic Industry Publishers 2003, 531 - 539 